\title{
El modelo de la economía social, solidaria y colaborativa en América Latina como un nuevo modelo de empresa
}

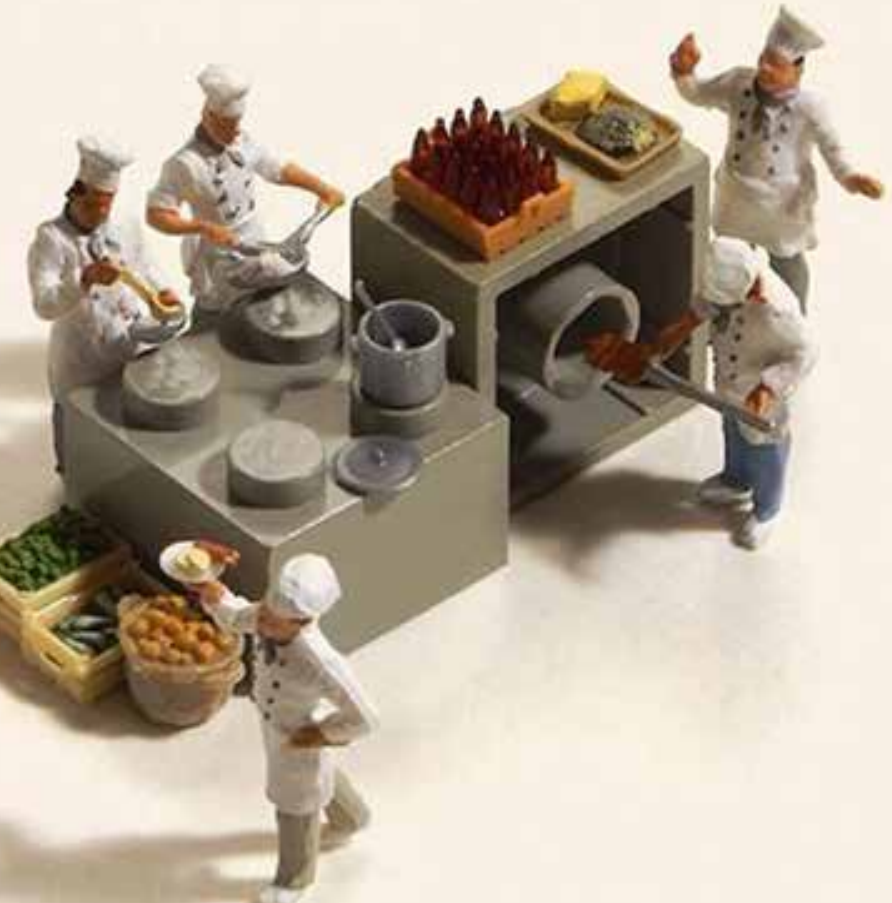

Por. Mtra. Haydee Beatriz Cartagena de Leiva Jefe de departamento y docente investigadora del Departamento de Administración de Empresa, UCA hcartagena@uca.edu.sv

Análisis basado en los resultados obtenidos en el trabajo de graduación de pregrado denominado: "Caracterización de las iniciativas de economía social y solidaria y colaborativa en América Latina a través de la investigación documental" para obtener el título de licenciatura en Administración de Empresas, elaborado por Brenda Lisette García Valle, Andrea Valeria Cabrera Ávila, María Hortencia Calderón Santamaría y Rolando Josué López Pérez; asesorado por la maestra Haydee Beatriz Cartagena.

\section{RESUMEN}

En un contexto marcado por una situación de pandemia por COVID-19 se ha reflexionado mucho sobre el modelo actual de las empresas, donde su eje principal ha sido la generación de ingresos a su máximo nivel y la acumulación de capital como el indicador principal de éxito, muchas veces, poniendo en riesgo el medio ambiente, la dignidad de las personas y el entorno que les rodea; en fin, arriesgando la sostenibilidad de la vida.

Ante esta situación es importante reflexionar sobre el actual modelo no sólo a nivel micro sino también macro, lo que significa tomar en cuenta aspectos como las políticas públicas y las relaciones que se establecen en las cadenas de valor en los diferentes rubros productivos.

El modelo de economía social, solidaria y colaborativa propone un nuevo modelo de empresa, siempre basada en la eficiencia, productividad y sostenibilidad financiera, pero con un elemento diferenciador: poner en el centro de sus decisiones a las personas. Muchas experiencias en América Latina y otros países del mundo nos muestran que es posible, especialmente, porque esto puede contribuir a la construcción de un nuevo modelo de desarrollo sostenible.

En este análisis se presentarán las características principales que definen esta propuesta, no sólo a través de los planteamientos teóricos existentes, sino también a través de las experiencias latinoamericanas que han mostrado resultados positivos, a nivel de las actividades productivas y también del entorno que les rodea.

\section{Palabras clave:}

Economía solidaria, capitalismo, personas, empresas, economía colaborativa, emprendimiento, cooperativismo.

El modelo de la economía social, solidaria y economía colaborativa (ESSC)

\section{La economía social y solidaria (ESS)}

Diversos autores convergen en que, tanto la economía social y la economía solidaria, tienen a la base el bien común y surgen a partir de acciones de intercambios de bienes y servicios, ya sea por otro bien o por dinero (Harguindeguy, 2008). 
La propuesta del CIRIEC (1990) citada por Chaves \& Monzón (2003) define a la ESS como:

"un conjunto de empresas impulsadoras de la economía que buscan la generación de riquezas para todos los grupos involucrados en esta realización, lo que implica que en la toma de decisiones todos participan, tanto de las responsabilidades como de los logros obtenidos, la distribución de ingresos es de forma equitativa, es decir enfocada al bienestar común, abonado a esto, vela por el cuidado del medio ambiente en general, donde el fin principal es ganar-ganar" (p. 10).

\section{La economía colaborativa}

Con relación a la definición académica de economía colaborativa no hay una que la describa, además, se dificulta su traducción específica del inglés al español. Sin embargo, se considera que la economía colaborativa está fundamentada en comunidades que definen el acceso de los bienes y servicios en función de necesidades concretas y no solo privilegiando la ganancia económica (Sastre \& Galiana, 2018).

La Comisión Nacional de los Mercados y la Competencia de España (CNMC) define a la economía colaborativa a través de múltiples elementos que, a su vez, han permitido mejoras y eficacia de las prácticas económicas que han sido parte de un modelo económico competidor y complementario al sistema capitalista por caracterizar los modelos de consumo y producción que no son orientados por un fin lucrativo (Sastre \& Galiana, 2018).

Además, se puede destacar que la economía colaborativa surge como una práctica que se vuelve cada vez más habitual para muchas personas, capaz de alcanzar un gran número de usuarios de manera rápida, que busca resolver necesidades y se orienta al mejor uso de la tecnología para el beneficio común.

\section{Surgimiento de la ESS en América Latina a través del cooperativismo.}

Los primeros indicios del funcionamiento de un nuevo modelo de empresa surgen en la primera mitad del siglo XIX, especialmente, en Argentina, Brasil, México y Venezuela. Es hasta las primeras décadas del siglo $X X$ que, de manera paulatina, se va desarrollando en el resto de los países a través de emprendimientos cooperativos (OIT, 2012).

Las cooperativas son empresas con principios y valores que se centran, principalmente, en la confianza y la cooperación, generando un carácter social como característica principal (Bretos, Diaz, Marcuello \& Marcuello, 2017).

\section{La Economía Social Solidaria y Economía Colaborativa} (ESSC)

El modelo de la ESSC es la fusión de los conceptos desarrollados anteriormente, ya que se describe como un modelo que busca el bienestar común y cuyo objetivo no es la búsqueda de riqueza a cualquier precio, sino que exista igualdad entre todas las personas que interactúan entre sí. Promueve la dinamización económica basada en un enfoque de derechos humanos y también busca una mejora social en la que todos los actores de la economía y su entorno sean beneficiados (Mendiguren \& Etxezarreta, 2015).

\section{Metodología}

El objetivo de la investigación fue caracterizar las iniciativas de economía social, solidaria y colaborativa en América Latina a través de una investigación documental sobre el desarrollo de este modelo en la región, esto permitió destacar las características y áreas más representativas del enfoque, lo que posibilitó obtener un panorama más amigable sobre el funcionamiento y estructuración de este modelo.

La metodología utilizada para la recopilación de la información fue de carácter cualitativo y documental; el principal instrumento para la recolección de información fue la revisión documental de estudios académicos, informes, libros, vídeos y documentales sobre las experiencias desarrolladas a lo largo de los años por proyectos de cooperación para el desarrollo y por diferentes cooperativas que también han contribuido al desarrollo del modelo. Así mismo, se planteó la identificación de iniciativas productivas que aplican el modelo de ESSC en América Latina, su perfil estándar y las buenas prácticas basadas en experiencias de la región.

La información fue consultada en diferentes bases de datos tales como EBSCO, la Organización Internacional del Trabajo (OIT), el metabuscador de la Universidad Centroamericana "José Simeón Cañas" (UCA), Google académico como base complementaria, libros, artículos de revistas científicas e investigaciones realizadas por la UCA, así como páginas web de organizaciones de cooperación que invierten en el desarrollo de iniciativas de ESSC y de las iniciativas ya existentes. 
Para asegurar la calidad de la información a revisar se realizaron filtros por medio de la plataforma Scimago para constatar que la información proviniera fuentes científicas confiables. En esta plataforma se verificaron dos criterios: (a) el cuartil en el que se encuentra, el cual indica la posición que tiene una revista en relación con respecto a las de su misma área y (b) el $\mathrm{H}$-Índex o índice, que mide la cantidad de citas que ha recibido cierto artículo científico.

\section{Características de la ESSC}

La ESSC consolida la gestión de las actividades económicas y solidarias con valores sociales en beneficio de todas las personas involucradas y de su entorno, genera una cultura democrática y se orienta a generar un desarrollo sostenible. Además, integra la búsqueda de la justicia social en los sistemas de producción, distribución y comercialización; tiene como una de sus principales características cuidar y respetar el medio ambiente, enriquecer la cultura, promover una relación donde todas las personas involucradas y su entorno puedan mejorar sus condiciones de vida a partir de su esfuerzo organizativo (Falcón \& Fuente, 2019).

La siguiente tabla presenta con mayor detalle, las principales características que describen el modelo.

Tabla 1. Características de ESSC

\section{Economía Social, Solidaria y Colaborativa}

Uso de tecnología para conectarse con diferentes grupos de personas o empresas, mediante el uso de bienes o servicios y la utilización de plataformas electrónicas.

\section{Uso de nuevas tecnologias por diferentes agentes que persiguen un objetivo común.}

Capacidad de trabajar en conjunto.

Existe un intercambio de información entre las diferentes personas y empresas siempre abonando a la idea principal de no lucrarse individualmente sino buscando bienestar común.

Ayuda a la eficiencia en la gestión empresarial, logrando la optimización de recursos mediante el uso de plataformas digitales.

Organización, en el sentido que permite a las personas reunirse con el fin de alcanzar ciertos objetivos comunes, desde el ámbito tanto económico como social.

El generar asociatividad en las comunidades para organizarse dentro de su territorio y asociarse con otras comunidades sin perder de vista la búsqueda del bienestar comunal.

La capacidad de autogestión que tienen las personas para poder auto administrar sus proyectos de los cuales seran beneficiados.

Fuente: (García, Cabrera, Calderón y López, 2020)

En resumen, se puede sintetizar que el modelo de la ESSC es la suma de los esfuerzos para lograr un fin común, parte de la organización y asociación de sus integrantes dirigida hacia el desarrollo de actividades productivas y sostenibles que contemplen a la persona como parte del proyecto económico y, además, tiene un componente social, con personal con conocimiento técnico o con la disposición para adquirirlo, pero también para ponerlo a disposición y así contribuir con el desarrollo propio y el de su entorno hacia el desarrollo sostenible.

Hoy en día, también se considera necesario que, para estar a la vanguardia, estas empresas incorporen en sus cadenas de valor las nuevas tecnologías de información y el uso de plataformas digitales, respetando y practicando los principios y valores que las definen. 


\begin{tabular}{|c|c|c|}
\hline Países & Legislación & Objetivo \\
\hline Honduras & $\begin{array}{l}\text { Ley del Sector Social de la } \\
\text { Economía (1985) }\end{array}$ & $\begin{array}{l}\text { Propone la economia social como el conjunto de empresas } \\
\text { u organizaciones formadas por trabajadores, quienes optan } \\
\text { por la propiedad social de los medios de producción y } \\
\text { establecen que el excedente económico sirva de medio para } \\
\text { mejorar el nivel de vida de sus miembros. }\end{array}$ \\
\hline Colombia & $\begin{array}{l}\text { Ley } n^{\circ} 454 \text { de la Economía } \\
\text { Solidaria. (1998). }\end{array}$ & $\begin{array}{l}\text { Establecen los principios y características de la economía } \\
\text { social y solidaria, así como las personas jurídicas que } \\
\text { califican en este tipo de economia. }\end{array}$ \\
\hline México & $\begin{array}{l}\text { Ley General de la Economia } \\
\text { Social y Solidaria (2007) (2011) }\end{array}$ & $\begin{array}{l}\text { Establece las reglas de organización, promoción, fomento } \\
\text { y fortalecimiento en un sistema eficaz que contribuya al } \\
\text { desarrollo social y económico, a la generación de empleos, } \\
\text { al fortalecimiento de la democracia y a la equidad de la } \\
\text { distribución del ingreso y la difusión de la propiedad. }\end{array}$ \\
\hline Ecuador & $\begin{array}{l}\text { Ley de economía popular y } \\
\text { solidaria de Ecuador (2011) }\end{array}$ & $\begin{array}{l}\text { Fortalece el sector para generar empleo e ingresos, pero que } \\
\text { nunca se habian tenido en cuenta hasta ahora. Además, de } \\
\text { establecer un marco legal para aquellos que la componen; } \\
\text { las cooperativas, las asociaciones y la comunidad. }\end{array}$ \\
\hline Venezuela & $\begin{array}{l}\text { Ley para el Fomento y } \\
\text { Desarrollo de la Economía } \\
\text { Popular Decreto N6130 (2008) }\end{array}$ & $\begin{array}{l}\text { Tiene como objetivo fortalecer, promover y proteger a las } \\
\text { entidades de carácter social. }\end{array}$ \\
\hline Bolivia & $\begin{array}{l}\text { Constitución de la republica } \\
\text { Art. } 311\end{array}$ & $\begin{array}{l}\text { Promueve el desarrollo de entidades solidarias, identifica } \\
\text { sus principios y características, tiene incluidos en sus } \\
\text { estatutos prácticas solidarias, democráticas y humanistas } \\
\text { en donde la autogestión es también un componente esencial. }\end{array}$ \\
\hline & $\begin{array}{l}\text { Ley de Economia Social y } \\
\text { Solidaria Provincia de Mendoza } \\
\text { (2012) Mendoza (2012) }\end{array}$ & $\begin{array}{l}\text { Define las entidades que pertenecen al modelo de Economia } \\
\text { Social y Solidaria, dictamina sus principios tales como: } \\
\text { solidaridad, cooperación y autoridad legítima, la apropiación } \\
\text { y disposición de recursos, etc. }\end{array}$ \\
\hline
\end{tabular}

Fuente: García, Cabrera, Calderón y López (2020, p.19-20 )

Por otra parte, han surgido algunas iniciativas de ley que han sido expuestas por Brasil y República Dominicana, quiénes están comenzando con el pero también se identificó que solo siete países de la región cuentan con este instrumento legal que ampara el funcionamiento, los apoyos y la inversión, pero que también incentiva al sector económico para la adopción de este modelo.
En el análisis del marco regulatorio de la ESSC en América Latina se identificó una legislación reciente

\section{Marco regulatorio de la ESSC existente}

Tabla 2. Legislación ESSC en América Latina 
Tabla 3. Iniciativa de ley ESSC en países de América Latina

\begin{tabular}{|c|c|c|}
\hline Países & \multicolumn{1}{|c|}{ Legislación } & \multicolumn{1}{c|}{ Objetivo } \\
\hline Brasil & $\begin{array}{l}\text { Proyecto Ley Economía } \\
\text { Solidaria (2010 }\end{array}$ & $\begin{array}{l}\text { Establece principios, objetivos y composición de la política, } \\
\text { crea el Sistema Nacional de Economia Solidaria que tiene } \\
\text { como objetivo de fomentar la Economía Solidaria y asegurar } \\
\text { el derecho al trabajo asociado y cooperativo. }\end{array}$ \\
\hline $\begin{array}{c}\text { República } \\
\text { Dominicana }\end{array}$ & $\begin{array}{l}\text { Proyecto de Ley República } \\
\text { Dominicana (2010) }\end{array}$ & $\begin{array}{l}\text { Pretende contribuir y modernizar la legislación dominicana, } \\
\text { teniendo un enfoque de implementar una pluralidad en el } \\
\text { sector económico al reconocer todas las formas de hacer } \\
\text { economía. }\end{array}$ \\
\hline
\end{tabular}

Fuente: García, Cabrera, Calderón y López (2020, p.20-21 )

\section{Vinculación de la ESSC con los ODS}

Existe una vinculación entre la ESSC y los Objetivos de Desarrollo Sostenible (ODS), ya que la esencia humana de este modelo aspira a contribuir a reducir las desigualdades y el rezago económico que existe en la sociedad actual (Luna, 2016).

Los ODS que tienen mayor relevancia en este modelo son:

- Objetivo 1 y 7: fin de la pobreza y trabajo decente y crecimiento económico, porque la ESSC busca el bienestar de todos los involucrados, generando un beneficio económico igualitario para todas las partes.

- Objetivo 10: reducción de las desigualdades, sin discriminación y buscando el bienestar común para hombres, mujeres, niños y niñas.

- Objetivo 13: acción por el clima, ya que parte muy importe de la ESSC es alcanzar una sostenibilidad ambiental.
Clasificación del modelo de ESSC en Latinoamérica Como ya se ha descrito, este modelo surge como resultado de la unión de tres tipos de economías: social, solidaria y colaborativa. Esto plantea una alternativa al modelo económico actual y pasa a uno que está centrado en las personas, su entorno, promueve la participación en la toma de decisiones y busca la sostenibilidad de la vida como objetivo principal, más que la acumulación de capital.

Es decir, es un modelo económico, social, medioambiental y colaborativo que busca responder a las necesidades de la población, aprovechando el sentido de pertenencia y buscando construir una identidad colectiva y de cooperación para el beneficio común y la dignificación de las personas.

En esta investigación se realizó una tipología de las iniciativas de economía social, solidaria y colaborativa, obteniendo tres tipos según tres categorías (A) Finalidad, (B) Estructura y (C) Sector, éstas a su vez se clasifican en subtipos, los cuales se describen en la siguiente ilustración:
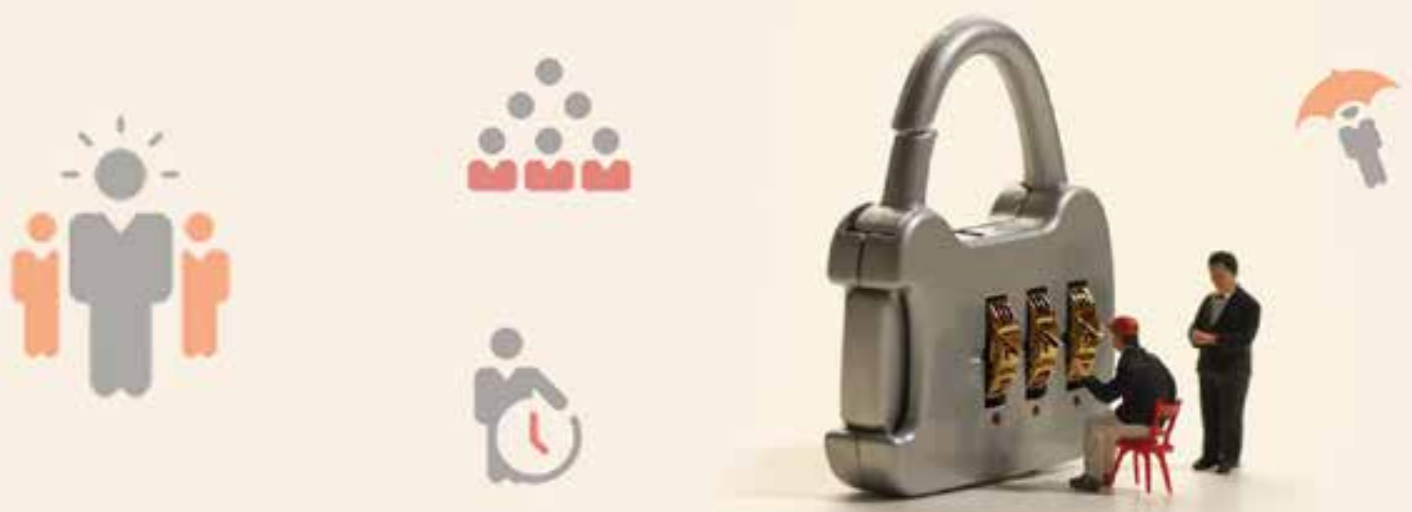
Ilustración 1. Tipos de ESSC

TIPOS DE ECONOMÍA SOCIAL, SOLIDARIA Y COLABORATIVA
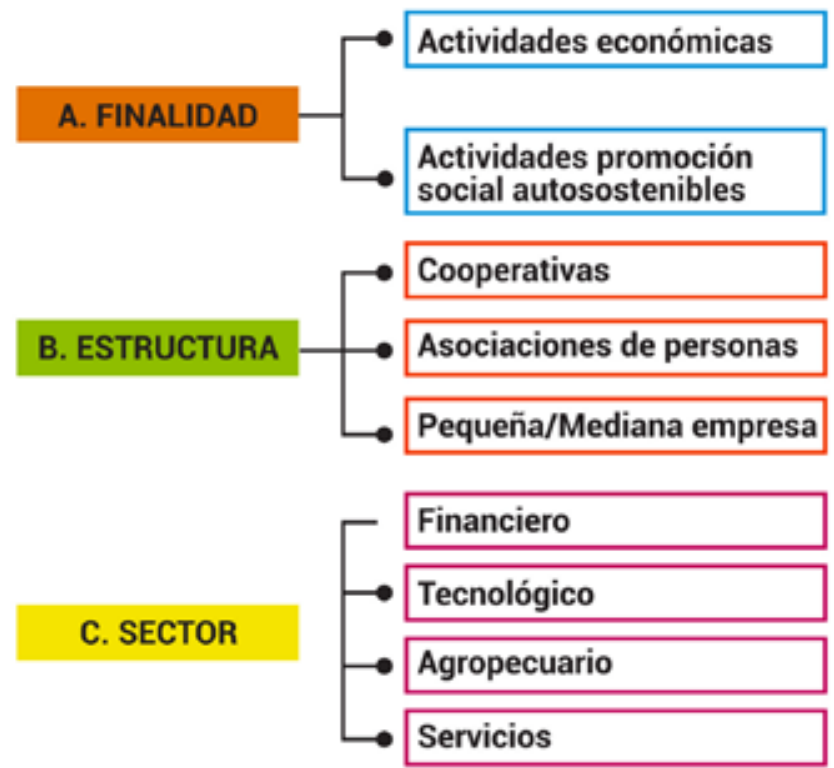

Fuente: García, Cabrera, Calderón y López (2020, p.35 )

\section{Perfil de ESSC en Latinoamérica}

A través de esta investigación y el análisis realizado, se ha propuesto el perfil de las empresas de ESSC que articula las características principales en la región de América Latina, en su mayoría, se diferencian de las empresas del modelo económico predominante, el cual está basado en la acumulación de capital como una prioridad. En la ilustración 2 se presentan las características del modelo de ESSC en la región latinoamericana.

Ilustración 2. Perfil de Economía social, solidaria y colaborativa (ESSC) en América Latina.

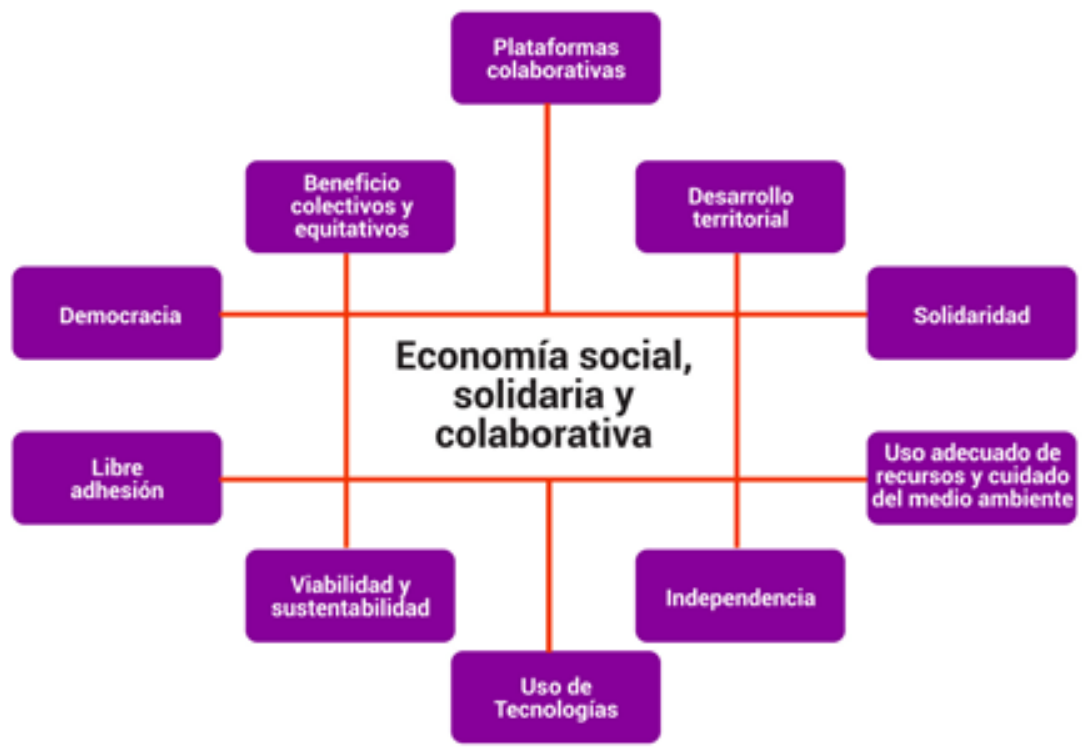

Fuente: García, Cabrera, Calderón y López (2020, p.48 ) 
Estas características se definen de la siguiente manera:

Plataformas colaborativas: conocidas como herramientas informáticas que faciliten y optimicen la comunicación e interacción entre las personas involucradas e interesadas en las iniciativas, así como participantes de la cadena de valor.

Desarrollo territorial: las ESSC buscan contribuir en una mejora en el bienestar, la calidad de vida y el desarrollo de la sociedad.

Solidaridad: todas las personas involucradas tienen la misma participación y se apoyan mutuamente buscando siempre el bienestar común, teniendo en cuenta que todas las personas están en el centro de las decisiones económicas.

Uso adecuado de recursos y cuido del medio ambiente: la ESSC vela por el adecuado uso de los recursos y lucha contra el cambio climático y la disminución de los impactos medioambientales.

Independencia: genera espacios para la toma de decisiones, es participativa y promueve la transparencia organizacional en su responsabilidad social y un gobierno libre.

Uso de tecnologías: incorpora la tecnología en su actividad diaria, de manera que permita ser más eficiente, pero que no se convierta en una herramienta de exclusión, sino que ofrezca soluciones de inclusión social, económica y medioambiental.

Viabilidad y sustentabilidad: la generación de ingreso se encuentra bajo la visión de desarrollo sostenible, la promoción de un desarrollo económico y social, que perduren en el tiempo.
Libre adhesión: inclusión de las personas que quieran formar parte, hay presente en la ESSC la promoción de un alto grado de interés y responsabilidad por parte de las personas y que éstas acepten los compromisos que implican la práctica de los principios y valores que se promueven.

Democracia: La ESSC promueve la participación informada, consensuada y orientada a la solución de los problemas de forma colectiva, respetando la diversidad de pensamiento.

Beneficios colectivos $y$ equitativos: promueve una cultura de igualdad y justicia, una adecuada comercialización de los productos generando ganancias y reparto equitativo de dichos recursos entre las personas que integran las iniciativas.

Este modelo propone una nueva forma de producir, consumir y distribuir, es una alternativa viable y sostenible para la satisfacción de las necesidades individuales y globales, contribuye al beneficio, no solo económico de las personas involucradas, sino también al beneficio social y medioambiental de la comunidad, poniendo la mirada hacia la construcción y consolidación del bien común de manera responsable.

\section{Retos que enfrenta la ESSC}

Como en todo modelo, siempre hay retos que superar, algunos que requieren mayor atención para generar condiciones adecuadas que permitan enfrentarlos y, en el mejor de los escenarios, minimizarlos o reducirlos. La siguiente ilustración tiene por objetivo enumerar los principales retos que han sido destacadas en las diversas experiencias y literatura investigada. 


\section{Ilustración 3. Retos que enfrenta la ESSC}

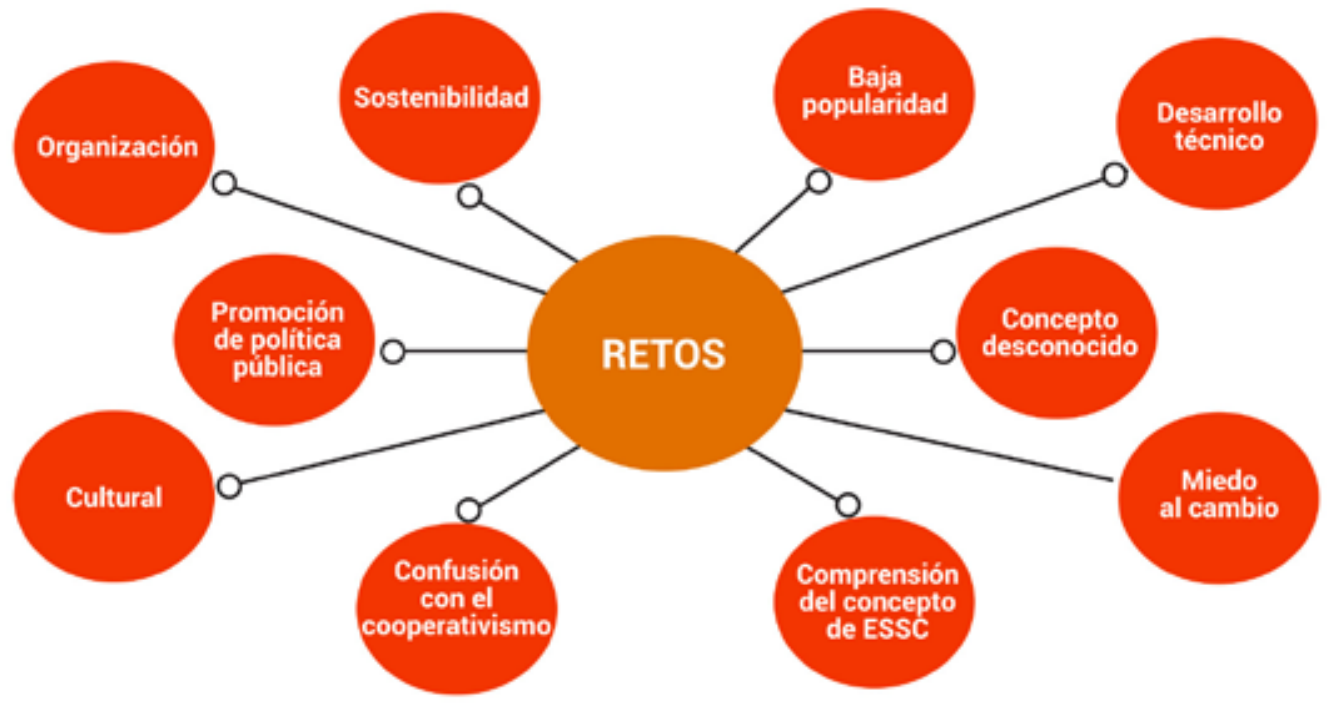

Fuente: García, Cabrera, Calderón y López (2020, p.63 )

Si bien es cierto, en la ilustración se presentan con detalle los retos identificados en este modelo, éstos también se pueden agrupar según sus principales actores:

1. En la población en general: la ESSC aún es desconocida para la mayoría de la población y la comprensión del concepto y del propósito del modelo puede ser difusa, por lo que el modelo tiene baja popularidad entre las personas. Además, el paradigma común entre las personas se basa únicamente en relaciones de intercambios y la acumulación del capital. Es decir, no se conocen alternativas.

2. En el sector empresarial: en primer lugar, se busca instalar la idea de que el cooperativismo es parte de una ESSC en su esencia y, en segundo lugar, se busca ayudar a comprender que se debe buscar la sostenibilidad económica y organizativa, además de promover procesos de organización comunitaria. Estos elementos implican grandes cambios en el sector empresarial, en sus propósitos, prácticas y paradigmas.

3. Con los tomadores de decisión: un reto bastante grande es encontrar programas de desarrollo e innovación tecnológica disponibles para empresas que practican el modelo de ESSC y la promoción de políticas públicas que generen contextos adecuados y las impulsen.

\section{Oportunidades que brinda el modelo de ESSC}

- Es un modelo integral con un nuevo enfoque de hacer economía, dándole la prioridad al bien común y a la mejora de la calidad de vida de las personas. Es un modelo que busca superar las brechas existentes en el modelo capitalista, principalmente, en la inclusión, participación de las personas y el respeto por el medio ambiente.

- Es un modelo que brinda mayores oportunidades a todas las personas, y que da consideración especial, a la población más vulnerable porque en todas las decisiones de las iniciativas económicas y sociales, el ser humano es el centro y no la acumulación de capital como en el modelo económico tradicional.

- Cuenta con diversas modalidades de organización, tales como cooperativas, fundaciones, asociaciones, empresas, canales de comercio electrónico e iniciativas de desarrollo.

- Las empresas enmarcadas en este modelo económico basan sus relaciones en el apoyo mutuo, la satisfacción de necesidades y en la colaboración; se busca el crecimiento y desarrollo de una sociedad más cercana a la empresa, pero también busca un crecimiento económico donde la dignidad y la calidad de vida de las personas sea lo fundamental. 
- Frente al desempleo y desaceleración económica que ya se empieza a ver a causa de la pandemia de COVID-19, así como el efecto que esto tendrá en el incremento de las desigualdades, este modelo ofrece una respuesta en términos de seguridad económica, social y ambiental, ofrece un trabajo articulado entre gobiernos, empresas y población enfocado en la priorización de recursos y atención de necesidades que permitan un desarrollo colectivo más que individual.

- Ante cualquier tipo de crisis económica, política, ambiental o sanitaria e modelo se vuelve una alternativa para la generación de formas de vida más inclusivas y sostenibles.

- Busca una distribución de los beneficios más justa e igualitaria para las personas involucradas; esto es la búsqueda del bien común, una distribución de los beneficios económicos que se base en cumplir los derechos universales y en la práctica de los principios y valores éticos que una sociedad requiere para su bienestar.

\section{Conclusiones}

La ESSC es un modelo inclusivo, integral y con un enfoque sistémico de la realidad donde las empresas se desarrollan. En el corto plazo puede ser que no genere grandes resultados económicos, pero con una buena gestión permite generar soluciones sostenibles para quienes integran tanto el sector empresarial como la sociedad.

Este modelo promueve una articulación entre los diversos sectores -tales como emprendedores, el de los gremios, cooperativas, entre otros - que permite crear sinergias tanto desde la cadena de valor, como hacia el desarrollo del entorno y la incidencia en las políticas públicas. En ese sentido, es importante promover un marco legal en los países de América Latina que aún no lo tienen, pero también a nivel regional, de manera que permita amparar y fortalecer el contexto para brindar condiciones que permitan a las empresas de ESSC desarrollarse.

Sabemos que el uso de tecnologías es fundamental en todos los aspectos de las empresas, pues en la actualidad se han vuelto una necesidad básica en la actividad empresarial e individual, estar a la vanguardia con esos temas, permite potenciar el desarrollo del sector empresarial que aplica este modelo.

Es necesario considerar los Objetivos de Desarrollo Sostenible (ODS) y destacar los efectos positivos que su integración genera, no sólo al interior de las empresas, sino también en sus alrededores. Con esto, se brinda la posibilidad de apoyar a las necesidades de las comunidades y poner como el centro de todas las decisiones a las personas.

Por otra parte, es importante involucrar a la academia para la generación de procesos de formación e investigación en el marco del modelo de ESSC para que brinde y difunda las principales características y beneficios del modelo, especialmente, en una situación de emergencia como la que vivimos actualmente. En este marco también es importante concentrar esfuerzos en generar mecanismos de difusión y sensibilización sobre la importancia y beneficios que ofrece este modelo económico, para influir de esta manera en las generaciones de nuevos profesionales y en el desarrollo de agentes innovadores y con alto compromiso social. 
Finalmente, considerar al modelo con un enfoque sistémico es primordial ya que genera articulación local, nacional, regional e internacional fortaleciéndose así el sector empresarial, sus relaciones y mecanismos de comercialización.

\section{Bibliografia:}

Bretos, I., Díaz-Foncea, M., Marcuello, C., \& Marcuello, C. (2018). Cooperativas, capital social y emprendimiento: Una perspectiva teórica. REVESCO. Revista De Estudios Cooperativos, 128, 76-98. https://doi.org/10.5209/REVE.59775

Falcón, C., \& Fuente, J. (2019). Mejorando el bienestar de la sociedad a través del cooperativismo de plataforma. CIRIEC- España, revista de Economía Pública, Social y Cooperativa, 95, 160-190. DOI: https://doi.org/10.7203/CIRIEC-E.95.12655

García, B. L., Cabrera, A. V., Calderón, M. H., \& López, R. J. (2020). El modelo de la economía social, solidaria y colaborativa en América

Latina a través de una investigación documental. San Salvador, El Salvador.

Harguindeguy, L. C. (2008). La economía social y solidaria. DEI, Departamento Ecuménico de Investigaciones

Luna, R. (2016). El vínculo de la ESS con los Objetivos de Desarrollo Sostenible - America Latina. RIPESS. Recuperado el 14 de Abril de 2020 de http://www.ripess.org/el-vinculo-de-la-ess-con-los-objetivos-de-desarrollo-sostenible-america-latina/?lang=es

Monzón, J., \& Chaves, R. (2016). Evolución reciente de la economía social en Europa. Comite Económico y Social Europeo. Obtenido de https://www.eesc.europa.eu/sites/default/files/files/qe04-17-875-es-n.pdf.

Pérez de Mendiguren, Juan Carlos, \& Etxezarreta, Enekoitz (2015). Sobre el concepto de economía social y solidaria: aproximaciones desde Europa y América Latina. Revista de Economía Mundial, (40),123-143. Disponible en: https://www.redalyc.org/ articulo.oa?id=866/86641407006

OIT. (2012). El cooperativismo en América Latina Una diversidad de contribuciones al desarrollo sostenible. Obtenido de https://www. ilo.org/wcmsp5/groups/public/---americas/---ro-lima/documents/publication/wcms_188087.pdf

Sastre, J., \& Galiana, M. (2018). La economía colaborativa: un nuevo modelo económico. CIRIEC - España, Revista de Economía Pública, Social y Cooperativa, 94, 219-250. Obtenido de https://doi.org/10.7203/CIRIEC-E.94.1210 